Article

\title{
Investigation of the Carrier Movement through the Tunneling Junction in the InGaP/GaAs Dual Junction Solar Cell Using the Electrically and Optically Biased Photoreflectance Spectroscopy
}

\author{
Sanam SaeidNahaei ${ }^{1}$, Hyun-Jun Jo ${ }^{1}$, Sang Jo Lee ${ }^{1}$, Jong Su Kim ${ }^{1, *}$, Sang Jun Lee ${ }^{2}$ and Yeongho Kim ${ }^{2}$ \\ 1 Department of Physics, Yeungnam University, Gyeongsan 38541, Korea; sanam.nahaei@yu.ac.kr (S.S.); \\ quantum@ynu.ac.kr (H.-J.J.); sjlee91@ynu.ac.kr (S.J.L.) \\ 2 Korea Research Institute of Standard and Science, Daejeon 34113, Korea; sjlee@kriss.re.kr (S.J.L.); \\ ykim172@kriss.re.kr (Y.K.) \\ * Correspondence: jongsukim@ynu.ac.kr
}

Citation: SaeidNahaei, S.; Jo, H.-J.; Lee, S.J.; Kim, J.S.; Lee, S.J.; Kim, Y. Investigation of the Carrier Movement through the Tunneling Junction in the InGaP/GaAs Dual Junction Solar Cell Using the Electrically and Optically Biased Photoreflectance Spectroscopy. Energies 2021, 14, 638. https:// doi.org/10.3390/en14030638

Received: 12 January 2021

Accepted: 22 January 2021

Published: 27 January 2021

Publisher's Note: MDPI stays neutral with regard to jurisdictional claims in published maps and institutional affiliations.

Copyright: (c) 2021 by the authors. Licensee MDPI, Basel, Switzerland. This article is an open access article distributed under the terms and conditions of the Creative Commons Attribution (CC BY) license (https:// creativecommons.org/licenses/by/ $4.0 /)$.

\begin{abstract}
For examining the carrier movements through tunnel junction, electrically and opticallybiased photoreflectance spectroscopy (EBPR and OBPR) were used to investigate the internal electric field in the InGaP/GaAs dual junction solar cell at room temperature. At InGaP and GaAs, the strength of $\mathrm{p}-\mathrm{n}$ junction electric fields $\left(\mathrm{F}_{\mathrm{pn}}\right)$ was perturbed by the external DC bias voltage and $\mathrm{CW}$ light intensity for EBPR and OBPR experiments, respectively. Moreover, the $\mathrm{F}_{\mathrm{pn}}$ was evaluated using the Fast Fourier Transform (FFT) of the Franz-Keldysh oscillation from PR spectra. In the EBPR, the electric field decreased by increasing the DC bias voltage, which also decreased the potential barrier. In OBPR, when incident CW light is absorbed by the top cell, the decrement of the $\mathrm{F}_{\mathrm{pn}}$ in the GaAs cell indicates that the photogenerated carriers are accumulated near the p-n junction. Photogenerated carriers in InGaP can pass through the tunnel junction, and the PR results show the contribution of the modification of the electric field by the photogenerated carriers in each cell. We suggest that PR spectroscopy with optical-bias and electrical-bias could be analyzed using the information of the photogenerated carrier passed through the tunnel junction.
\end{abstract}

Keywords: InGaP/GaAs dual junction solar cell; optically biased photoreflectance; electrically biased photoreflectance; tunnel junction

\section{Introduction}

Semiconductors play an important role in solar cell technology, where the solar cell's main parameter is high efficiency. The reported efficiency of the single-junction solar cells is low, and the multi-junction solar cells (SC) represent the most efficient approach to photovoltaic energy conversion, reporting an efficiency of around $40 \%$. The multi-junction structure is designed based on the selective absorption of different significant parts of the solar spectrum. Different absorbers in cells improve the photogeneration and collection of minority carriers and reduce energy losses related to hot carriers' thermalization [1]. As the illuminated light enters the cell, the short and long wavelengths are absorbed in the top and bottom cells, respectively.

In a multi-junction solar cell, the cells are interrelated by the tunnel junctions to align the Fermi level. The tunnel junctions should have less electrical resistivity, a high optical transmission, and a high peak tunneling current density. Without the tunnel junction, the p-region of the top cell and n-side of the bottom cell directly connected, and the p-n junction in the opposite direction appears between cells. Therefore, the photo-voltage would be decreased. In theory, the generated electrons in the top cell and holes in the bottom cell will recombine in the tunnel junction, while the generated holes and electrons in the top and bottom cell will go through the load to form a complete current loop [2,3].

InGaP, which has a wide bandgap of about $1.9 \mathrm{eV}$ and GaAs with $1.42 \mathrm{eV}$ bandgap energy, are suitable for the top and bottom cells of highly capable multi-junction solar 
cells. The combination of the InGaP (top cell) and GaAs (bottom cell) has around 40\% efficiency. The lattice parameters and photogeneration current of top and bottom cells can be coordinated when two cells consent together by the tunnel junction [3]. However, the lattice and current mismatch are two critical factors that lower the conversion efficiency of multi-junction solar cells. In the InGaP/GaAs dual junction structure, both lattice parameters and photogeneration current can be matched [4]. The whole current of the multi-junction solar cells series structure is bottlenecked by the cell with the smallest current. Therefore, currents from all cells should be matched as much as possible to control the electric power loss. One method to analyze the current matching of multijunction SC is studying the internal electric field in each cell's p-n junctions. It can provide information about the carriers' movement generated by the illuminated light through the tunnel junction to improve the current matching.

Under illumination in the solar cell, the photogenerated carriers can be capably divided by junction electric fields and transported as a photocurrent. Thus, the depletion region width and the internal electric field's strength is affected by generated electrons and holes. Therefore, the junction electric field related to the SC efficiency is an important material parameter of $\mathrm{p}-\mathrm{n}$ junction SC. Under light illumination, the $\mathrm{p}-\mathrm{n}$ junction electric field $\left(\mathrm{F}_{\mathrm{pn}}\right)$ is varied because of the photovoltaic effect. The photovoltaic effect defines a photogenerated carrier effect on the internal electric field in the $\mathrm{p}-\mathrm{n}$ junction. The photogenerated carriers induced an electric field in the opposite direction of the built-in electric field, and the total internal electric field is decreased. By increasing the photogenerated carrier density, the $\mathrm{F}_{\mathrm{pn}}$ may be decreased due to the photovoltaic effect. In the multi-junction solar cells, the generated carriers are passed to another cell through the tunnel junction and affect the electric field. Therefore, the photovoltaic effect on the internal electric field is an important parameter in investigating the current matching in multi-junction solar cells [5].

Electromodulation spectroscopy (EM), primarily the PR has been broadly used to examine semiconductor structure [6]. In the PR, the basic principle is to take the optical spectrum's derivative for a modulation parameter, which is an electric field [7]. Therefore the PR spectroscopy is a useful method to investigate the optical characteristic of SC. The PR spectroscopy is sensitive to the surface and interface electric field, and the FKO oscillation appears for the energies above the bandgap. The period of the FKO is related to the strength of the internal electric field in the sample. At the same time, the FKO could investigate the internal electric field of semiconductors in the PR spectrum. As a vital non-destructive method with high sensitivity and high spectral resolution, the PR spectroscopy has drawn ample courtesy in the experimental investigations of multi-junction semiconductors [8]. The PR reported a few experimental results to explore the InGaP/GaAs optical properties. However, the experimental observation of the tunnel junction impact in InGaP/GaAs has not been much focused on in many studies.

In this study, the electrical and optical biased photoreflectance (EBPR and OBPR) is used to examine the tunneling effect on the carrier density movement in this study's dual junction solar cells. The EBPR spectrum is recorded by measuring the PR amplitude as a function of the DC bias voltage at the fixed pump beam intensity, and the OBPR spectrum is recorded by measuring the PR spectra as a function of the CW light intensity. The DC bias voltage and CW light's internal electric field variation are measured to investigate the tunnel junction effect on the carrier density.

\section{Experiment}

The dual junction solar cell structure consists of an $\operatorname{In}_{0.48} \mathrm{Ga}_{0.52} \mathrm{P}$ top cell and a GaAs bottom cell with n-on-p polarity interconnected with a GaAs tunnel diode. Figure 1 shows the InGaP/GaAs dual junction solar cell's schematic structure with energy bandgaps of $1.9 \mathrm{eV}$ for the top cell and $1.42 \mathrm{eV}$ for the bottom cell. In a tandem configuration, the InGaP top cell absorbs shorter wavelengths $(\lambda<\sim 650 \mathrm{~nm})$ of solar radiation, while the GaAs bottom cell absorbs the longer wavelengths $(\sim 650<\lambda<\sim 870 \mathrm{~nm})$. The top and bottom cells have a back surface field (BSF) layer of $\mathrm{p}^{+}-\mathrm{InGaP}$, which forms a potential barrier for 
the minority carrier (electron), reducing rear surface recombination. As a window layer with a higher bandgap than the base layer, $\mathrm{n}^{+}-\mathrm{Al}_{0.53} \mathrm{In}_{0.47} \mathrm{P}$ and $\mathrm{n}^{+}-\mathrm{In}_{0.48} \mathrm{Ga}_{0.52} \mathrm{P}$ layers are introduced in the top and bottom cells to suppress the front surface recombination, respectively. A highly doped GaAs tunnel junction is embedded between the top and bottom cells to electrically and optically interconnect them [9].

\begin{tabular}{|c|c|c|}
\hline $50 \mathrm{~nm}$ & $\mathbf{n}^{+}$GaAs Contact & $2 \mathrm{E} 18 \mathrm{~cm}^{-3}$ \\
\hline $30 \mathrm{~nm}$ & $\mathbf{n}^{+} \mathbf{A} \mathbf{1}_{0.53} \mathbf{I n}_{0.47} \mathbf{P} \quad$ Window & $4 \mathrm{E} 17 \mathrm{~cm}^{-3}$ \\
\hline $50 \mathrm{~nm}$ & $\begin{array}{c}\mathbf{n}^{+} \mathbf{I}_{0.48} \mathrm{Ga}_{0.52} \mathbf{P} \\
\text { Emitter }\end{array}$ & $2 \mathrm{E} 18 \mathrm{~cm}^{-3}$ \\
\hline $0.65 \mu \mathrm{m}$ & $\begin{array}{c}\mathbf{p} \mathbf{I n}_{0.48} \mathbf{G a}_{0.52} \mathbf{P} \\
\text { Base }\end{array}$ & $7 \mathrm{E} 16 \mathrm{~cm}^{-3}$ \\
\hline $40 \mathrm{~nm}$ & $\mathbf{p}^{+} \mathbf{I n}_{0.48} \mathbf{G a}_{0.52} \mathbf{P} \quad$ BSF & $2 \mathrm{E} 18 \mathrm{~cm}^{-3}$ \\
\hline $15 \mathrm{~nm}$ & $\mathbf{p}^{++}$GaAs TJ & $>1 \mathrm{E} 19 \mathrm{~cm}^{-3}$ \\
\hline $15 \mathrm{~nm}$ & $\mathrm{n}^{++} \mathrm{GaAs}$ TJ & $>1 \mathrm{E} 19 \mathrm{~cm}^{-3}$ \\
\hline $50 \mathrm{~nm}$ & $\begin{array}{c}\mathbf{n}^{+} \mathbf{I n}_{0.48} \mathbf{G a}_{0.52} \mathbf{P} \\
\text { Window } \\
\end{array}$ & $2 \mathrm{E} 18 \mathrm{~cm}^{-3}$ \\
\hline $600 \mathrm{~nm}$ & $\mathbf{n}^{+} \mathrm{GaAs}$ Emitter & $2 \mathrm{E} 18 \mathrm{~cm}^{-3}$ \\
\hline $3.0 \mu \mathrm{m}$ & p GaAs Base & $1 \mathrm{E} 17 \mathrm{~cm}^{-3}$ \\
\hline $50 \mathrm{~nm}$ & $\mathbf{p}^{+} \mathbf{I n}_{0.48} \mathbf{G a}_{0.52} \mathbf{P} \quad$ BSF & $2 \mathrm{E} 18 \mathrm{~cm}^{-3}$ \\
\hline $200 \mathrm{~nm}$ & $\mathrm{p}^{+} \mathrm{GaAs}$ Contact & $4 \mathrm{E} 18 \mathrm{~cm}^{-3}$ \\
\hline & p-GaAs Sub. $350 \mu \mathrm{m}$ & \\
\hline
\end{tabular}

(a)

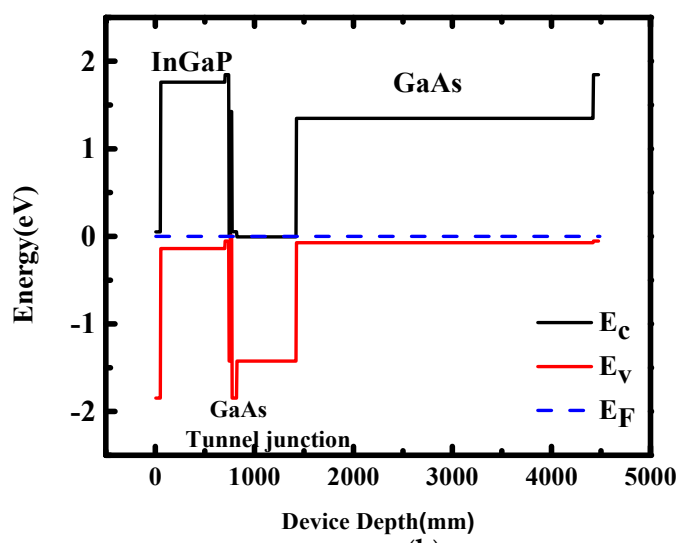

(b)

Figure 1. InGaP/GaAs dual junction solar cell: (a) layer structure and (b) schematic energy band diagram.

The solar cell structure was epitaxially grown on $(001) \mathrm{p}^{+}$-GaAs substrates using metalorganic chemical vapor deposition (MOCVD) in a Veeco D180 reactor. Trimethylaluminum (TMAl), trimethylgallium (TMGa), and trimethylindium (TMIn) were used as precursors of the group III elements. As precursors of the group V elements, high-purity arsine $\left(\mathrm{AsH}_{3}\right)$ and phosphine $\left(\mathrm{PH}_{3}\right)$ were used. Disilane $\left(\mathrm{Si}_{2} \mathrm{H}_{6}\right)$ and diethylzinc (DEZn) were used for ntype Si doping and p-type $\mathrm{Zn}$ doping. The MOCVD growth process was conducted under an optimum growth condition of $600{ }^{\circ} \mathrm{C}$ for the top and bottom cells and $540{ }^{\circ} \mathrm{C}$ for the GaAs tunnel diode, with a growth rate of $1 \mu \mathrm{m} / \mathrm{hr}$, and a V/III ratio of 130 . The as-grown 
structure was processed into solar cell devices with two terminals using a conventional photolithography technique. A wet etch process using citric acid-based solutions was applied to electrically isolate each solar cell device with a size of $1 \times 1 \mathrm{~mm}^{2}$. AuZn/Ni/Au and $\mathrm{Ge} / \mathrm{Au} / \mathrm{Ni} / \mathrm{Au}$ metals were deposited by an electron beam evaporation method for the p-type bottom and n-type top contacts of the solar cells, respectively, followed by a rapid thermal annealing process for ohmic contact formation.

The InGaP/GaAs dual junction solar cell's efficiency is around $10.69 \%$ under 1 sun AM 1.5G illumination. In this condition, the measured open-circuit voltage $\left(\mathrm{V}_{\mathrm{oc}}\right)$ is around $1.742 \mathrm{~V}$, and the short circuit density $\left(\mathrm{J}_{\mathrm{sc}}\right)$ is $7.47 \mathrm{~mA} / \mathrm{cm}^{2}$.

The $532 \mathrm{~nm}$ laser (Diode-Pumped Solid State laser) was used in the PR spectrum measurements as the modulation light. The monochromatic light was performed as a probe beam, and reflected light was detected by a GaAs detector. The lock-in amplifier gives the differential reflectance in a similar way as the standard PR measurements. However, in the EBPR measurements, the appropriate forward and reversed biased voltage $(-0.6 \sim 0.6 \mathrm{~V})$ was externally applied to the region of interest by a function generator at $300 \mathrm{~K}$. The externalbias voltage dependence on the built-in electric field is measured by the FFT analysis of the Franz-Keldysh oscillation (FKO). To apply the optical bias $\left(0 \sim 7400 \mathrm{~mW} / \mathrm{cm}^{2}\right)$ in the OBPR measurements, we employed the $404 \mathrm{~nm}$ laser diode in our PR experimental setup at $300 \mathrm{~K}$. The external bias light dependence of the built-in electric field was obtained utilizing the FFT method. Figure 2 shows the (a) Electrically Biased Photoreflectance (EBPR) and (b) Optically Biased Photoreflectance (OBPR) spectroscopy setup.

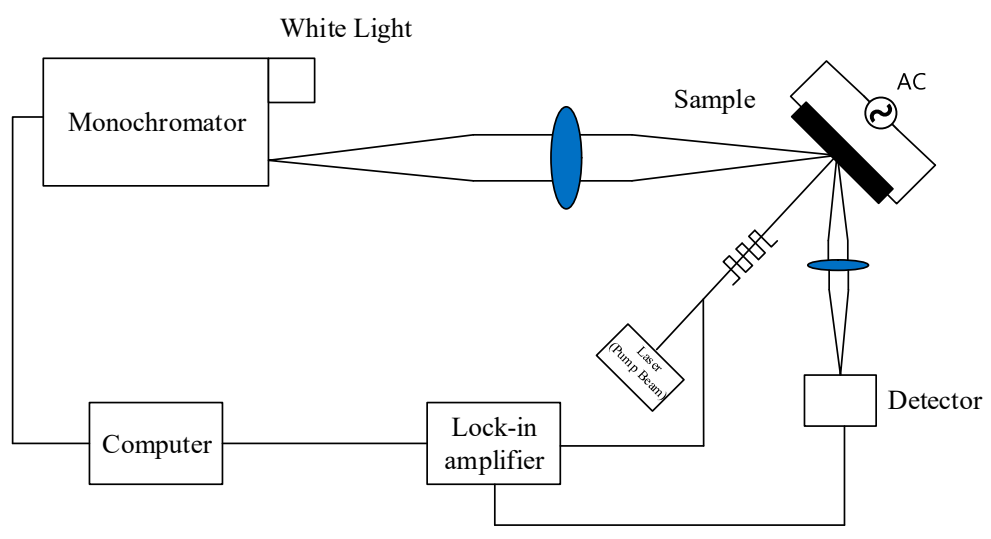

(a)

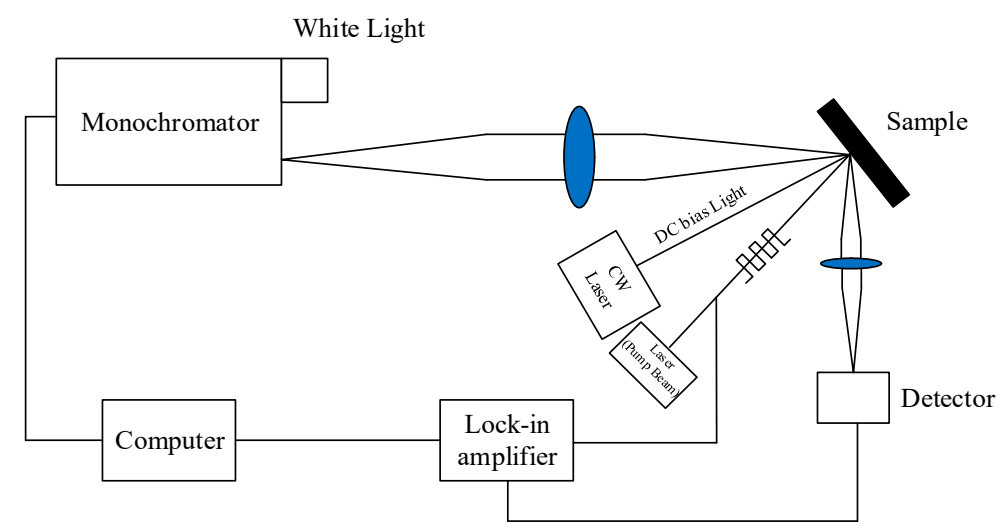

(b)

Figure 2. Schematic diagram of the experimental setup for (a) Electrically Biased Photoreflectance (EBPR) and (b) optically biased photoreflectance (OBPR) at room temperature. 


\section{Results and Discussion}

The PR spectra of the InGaP/GaAs solar cell is shown in Figure 3. The PR spectrum contains the InGaP and GaAs band-to-band transition $\left(\mathrm{E}_{\mathrm{gInGaP}}=1.9 \mathrm{eV}\right.$ and $\left.\mathrm{E}_{\mathrm{gGaAs}}=1.42 \mathrm{eV}\right)$ and Franz-Keldysh Oscillations (FKO). At room temperature, the FKO oscillation appears in the PR spectra above the InGaP and GaAs bandgap energy because of the surface and interface electric field, as shown in Figure 3. The Fast Fourier Transform (FFT) method was performed on the PR spectrum to recognize the FKO components in the frequency domain $\left(\mathrm{eV}^{3 / 2}\right)$, as shown in the inset figure.

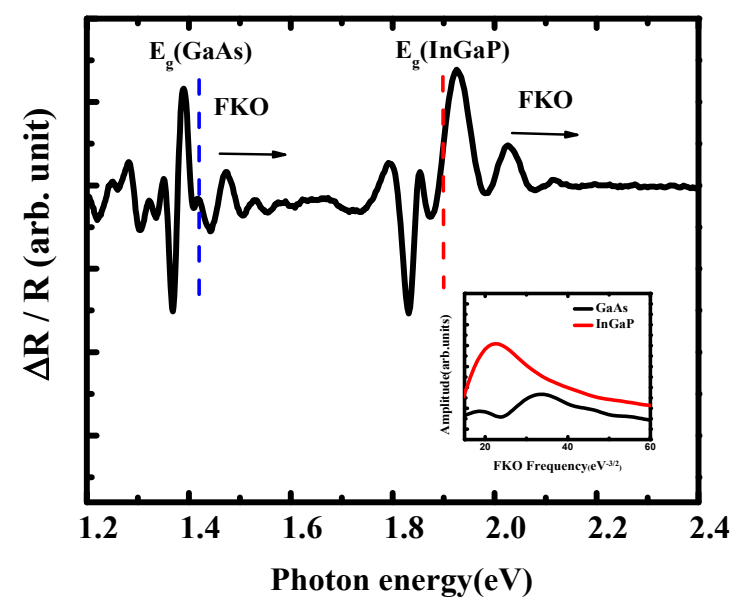

Figure 3. The PR spectra of InGaP/GaAs dual junction solar cell at room temperature.

In the high-field region, the oscillatory behavior of the FKO's is described by Equation [10]:

$$
\frac{\Delta R}{R}=\cos \left\{\frac{4}{3}\left[\frac{\left(E-E_{0}\right)^{3 / 2}(2 \mu)^{1 / 2}}{e \hbar F}\right]+\frac{\pi(d-1)}{2}\right\}
$$

where

$$
F=\frac{2}{3 \pi}(2 \mu)^{1 / 2} \frac{1}{e \hbar f_{F F T}}
$$

where the $E_{0}$ is the bandgap energy, $d$ is the dimensionality of the critical point, $e$ is the charge of an electron, $\hbar$ is Planck's constant, $f_{F F T}$ is the frequency of FKOs and $F$ is the electric field strength and $\mu$ is the reduced mass in the direction of $F$. To drive the electricfield strength, estimating the oscillating period $f_{F F T}$ of the FKO is done utilizing the FFT method. The period of the FKO reflects only the strength of the internal electric field as described in Equation (2), and it contains no information on its sign or direction [11].

Generally, under illumination, the space charge field in the depletion region drifted the generated electrons and holes in opposite directions in the steady-state. These separated excess carriers induce an internal electric field in the opposite direction to the space-charge field.

Thus, the depletion region's width and the space charge field's strength are changed by the photogenerated induced electric field [10]. The $\mathrm{F}_{\mathrm{pn}}$ is affected by the carrier density variation, and the $\mathrm{F}_{\mathrm{pn}}$ behavior is reflected in the PR spectra, especially FKO's, because the FKO is directly associated with interface electric field strength in the sample [12].

The PR spectra were measured using the $532 \mathrm{~nm}$ laser as the pump beam with energy $(2.33 \mathrm{eV})$ higher than the InGaP band gap energy $(1.9 \mathrm{eV})$. Therefore, the photons typically are absorbed by InGaP, and a relatively less amount of photons are transmitted and absorbed by the GaAs. Thus, the internal electric field is changed and decreased due to the photovoltaic effect. The frequency of oscillations in the FFT result is inversely related to the p-n junction's electric field, and the values of $\mathrm{F}_{\mathrm{pn}}$ related to the $f_{F F T}$ were 135.34 and $74.40 \mathrm{kV} / \mathrm{cm}$ for InGaP and GaAs, respectively. 
In the multi-junction SC, the tunnel junction was a diode made of a wide bandgap and highly doped material. This decreased the depletion region length, and therefore, the electron and holes could easily tunnel through. With a tunnel-junction structure, the device's current depends on electron transport on one side of the junction and hole transport on the other. By changing the carrier density, the internal electric field, which is an important parameter of the SC, is affected. Therefore, to investigate the SC properties and efficiency, determining the tunnel junction effect on the carrier density and internal electric field of cells is important [12].

The InGaP/GaAs dual junction SC operation under a DC bias voltage was performed using DC biased photoreflectance spectroscopy (EBPR) to investigate the effect of carrier movement through tunnel junction on the electric field. The results are shown in Figure 4.

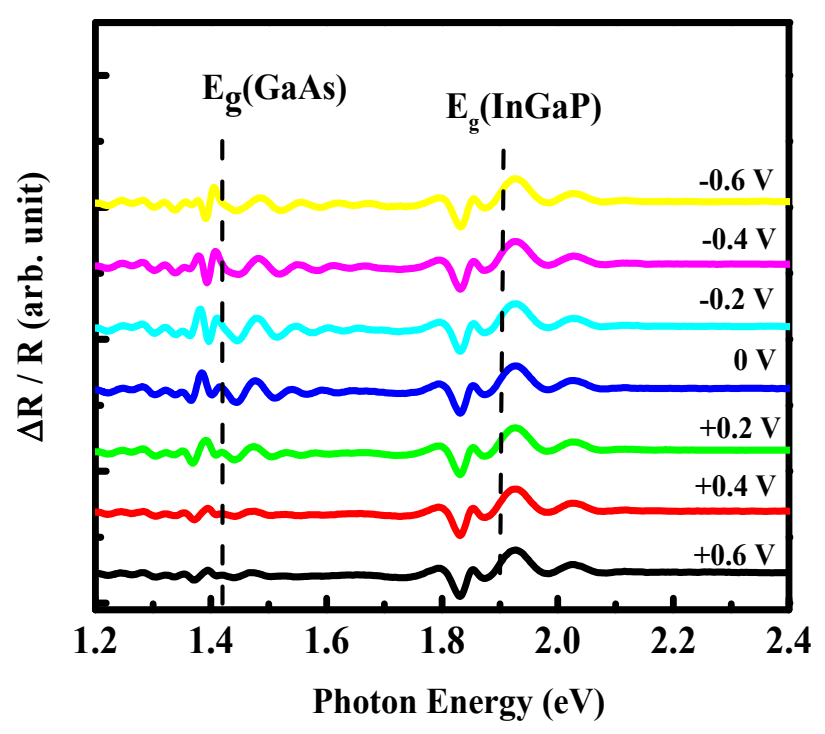

Figure 4. The photoreflectance spectra of InGaP/GaAs dual junction solar cell under zero, 0.2, 0.4, and $0.6 \mathrm{~V}$ of the forward and reverse DC bias voltage at $300 \mathrm{~K}$.

The DC bias voltage was applied using a digital function generator, and the DC bias voltage increased from $-0.6 \mathrm{~V}$ to $+0.6 \mathrm{~V}$. The PR spectra consisted of InGaP and GaAs band-to-band transition and FKO oscillations above the bandgap energies. The signals have a typical third derivate functional form (TDFF), but the amplitude of the PR signals and the Franz-Keldysh oscillations is changed when using the DC bias voltage. The shape and period of FKOs can be changed by altering the induced electric field by applying the DC bias voltage. By increasing the DC bias voltage, the peaks of the FKO shift to the left, and the period of the FKOs is decreased. The FKO components in the frequency domain are investigated using the FFT analysis on the PR spectrum. As described, the oscillation frequency is proportional to the strength of the internal electric field in the $p-n$ junctions.

The internal electric fields are calculated using the FFT analysis, which is exhibited in Figure 5. While the DC bias voltage was increased from -0.6 to $+0.6 \mathrm{~V}$, the FKO oscillations increased, and the value of $F_{p n}$ reduced from 142.3 to $130.3 \mathrm{kV} / \mathrm{cm}$ and 97.1 to $62 \mathrm{kV} / \mathrm{cm}$ for InGaP and GaAs cells, respectively.

Generally, if a suitable forward bias voltage is applied to the two ends of the p-n junction, it can supply free electrons and holes with the additional energy they require to cross the junction as the width of the depletion layer around the p-n junction decreases [13]. Decrement of the width of the depletion region and movement of the carrier to the $\mathrm{n}$ and $p$ side of the junction enhanced the photogenerated induced electric field in the opposite direction of the built-in electric field. This means that the total internal electric field was reduced. Additionally, applying a reverse bias voltage results in the free charges being pulled away from the junction resulting in the increased depletion layer width. Hence, 
in the InGaP and GaAs cells, an increment of the voltage increased the FKO frequency. Therefore, based on the description, the internal electric field decreased and the bias voltage enhanced, owing to reducing the potential barrier of the top and bottom of cells. Figure 5 shows the internal electrical field of InGaP and GaAs with various DC bias voltages. The results show that the decrement of the internal electric field in GaAs and InGaP is mostly due to the external bias voltage's potential barrier variation. Furthermore, the generated carrier also affects the $F_{p n}$ variation [14].

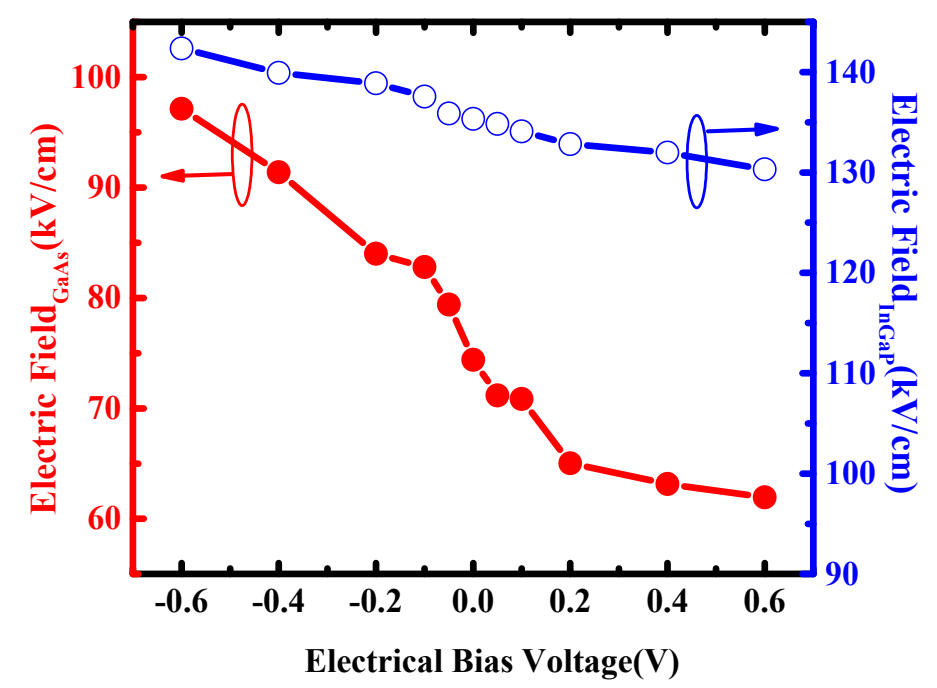

Figure 5. The internal electric field $\left(\mathrm{F}_{\mathrm{pn}}\right)$ of InGaP (open circle) and GaAs (solid circle) versus electrical DC bias voltage at $300 \mathrm{~K}$.

As described previously, the pump beam is absorbed mostly by the InGaP and a few photons are absorbed by the GaAs. Therefore, the photogenerated carriers in cells affect the internal electric field strength. Resultantly, the internal electric field decreased due to the photovoltaic effect in both cells. The $532 \mathrm{~nm}$ laser's input power was kept at $100 \mathrm{~mW} / \mathrm{cm}^{2}$, and the generated carrier density was constant during the measurements. This means that the voltage variation affected the potential barrier and internal electric field strength in the EBPR measurement.

Additionally, investigating the $\mathrm{F}_{\mathrm{pn}}$ in cells showed that the GaAs cell's decrement rate by the DC bias voltage was higher compared to the InGaP cell. Generally, the built-in potential of the $\mathrm{p}-\mathrm{n}$ structure depends on the intrinsic carrier and doping concentration. Therefore, the built-in potential in the GaAs is low. The small potential height indicates that under the same applied voltage, the variation of the potential barrier in GaAs is higher as compared to the InGaP. This indicates that due to the potential barrier's variation, more carriers may move and drift in the p-n junction in GaAs, and the external DC bias voltage comparably decreases the internal electric field strength.

In addition, in the PR measurements, the carrier effect is not negotiable because the probe beam effect is very small. By considering the internal electric field in the p-n junctions of cells, the carrier density is caused by the electrical bias voltage, and it is represented by the following Equation [14].

$$
E=\frac{Q}{\varepsilon_{s} \varepsilon_{0} A}=\frac{\sigma_{b i}}{\varepsilon_{s} \varepsilon_{0}}
$$

where, $\mathrm{E}$ is the electric field created by the bias voltage, $\mathrm{Q}$ is the quantity of electric charge, $A$ is the area of the sample, $\varepsilon_{\mathrm{s}}$ is the dielectric constant of InGaP and GaAs $\left(\varepsilon_{\text {InGaP }}=11.8\right.$, $\left.\varepsilon_{\mathrm{GaAs}}=12.85\right), \varepsilon_{0}$ is the permittivity of free space and $\sigma_{\mathrm{bi}}$ is carrier density caused by the electrical bias voltage. Figure 6 shows the number of carrier density contribution to the photovoltaic effect. The results show that the carrier's density, which contributes to the 
photovoltaic effect, in the GaAs is higher than InGaP. Meanwhile, in both cells, the carrier density number increased by increasing the reversed bias voltage.

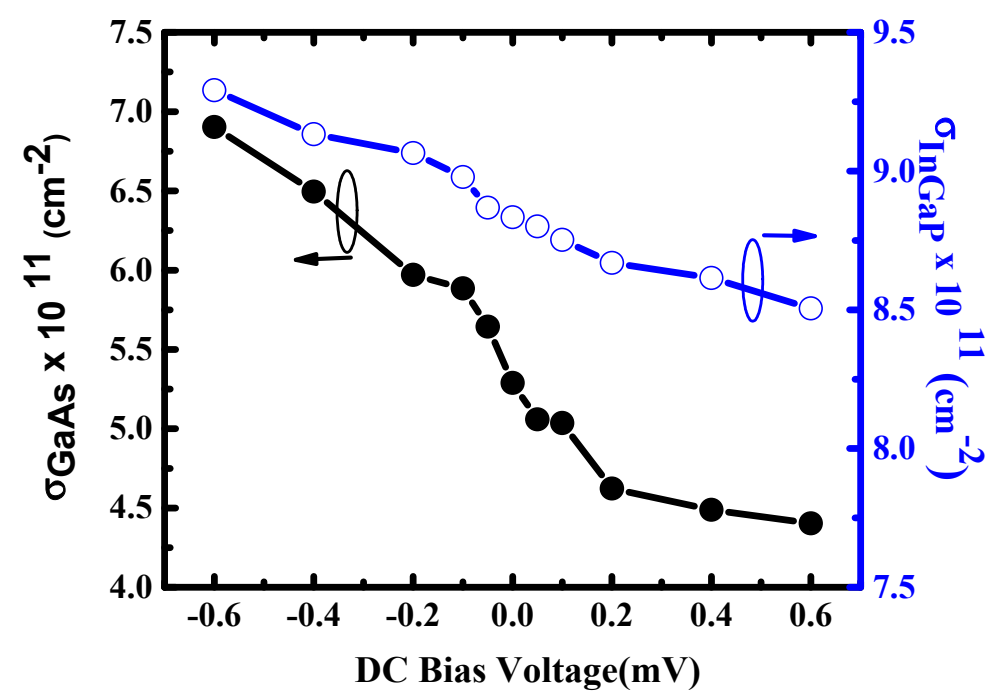

Figure 6. The number of carrier density caused by electrical bias voltage in InGaP (open circle) and GaAs (solid circle) as a function of the DC bias voltage at $300 \mathrm{~K}$.

In addition, the decrement of the internal electric field under reversed bias voltage is higher than that for the forward bias voltage. The p-n junction region acts as the electrode, and the depletion region of the p-n junction acts as the dielectric of the capacitors. Therefore, the multi-junction solar cell performs like the series capacitor. When the bias voltage is applied to the capacitor, the carriers reach the electrodes of capacitors, and a large number of charge carriers are accumulated in the electrodes [15]. These carriers establish the electric field between two electrodes because they could not move there. The capacitance is inversely dependent on the distance between two plates and directly proportional to the electrodes' size. Generally, in the p-n junction, two types of capacitance take place; transition and diffusion capacitance. For the reversed bias voltage, the carrier's large number moved to the $\mathrm{p}$ and $\mathrm{n}$ side by increasing the voltage, and the depletion region width increased, whereas the size of the $\mathrm{p}$ and $\mathrm{n}$-type region (plates) decreased. Therefore, the $p$-n junction with a wide depletion region and a small $p$ and n-type region will store a small electric charge. Therefore, the reverse bias p-n junction's capacitance decreases with voltage increment, while the potential and internal electric field variation are increased [16].

To investigate the photogenerated carrier movement through tunnel junction effect in InGaP/GaAs dual junction SC in more detail, we measured the Optical Biased photoreflectance (OBPR) in the InGaP/GaAs dual junction solar cell. In these measurements, the $404 \mathrm{~nm}$ laser was used as an incident CW light source at room temperature. The power dependence of the optical CW light PR spectra is shown in Figure 7. The CW light energy of $3.06 \mathrm{eV}$ will only generate the EHP in InGaP. The pump beam with an energy of around $2.3 \mathrm{eV}$ is also absorbed by cells. The carriers generated by the pump beam were located in both InGaP and GaAs. Previously we described that the InGaP mostly absorbs the pump beam, and the GaAs can absorb the small part of photons. Therefore, the carrier density generated by the pump beam in $\mathrm{InGaP}$ is higher than $\mathrm{GaAs}$. The pump beam intensity is kept constant, and the generated carrier density can be kept constant during the measurements. At the same time, the photogenerated carriers by the CW light are located in the InGaP. Therefore, the internal electric field in the InGaP is modulated by the photogenerated carrier within the InGaP, but not the GaAs cell. Thus, the PR signal is deformed by increasing the $\mathrm{CW}$ light intensity in both; InGaP and GaAs. This means that the internal electric field is modulated in cells. In the multi-junction solar cell, the carriers can pass to other cells through the tunnel junction. Therefore, generated carriers by the $\mathrm{CW}$ beam in the top cell can pass through the tunnel junction and change the carrier's density 
in the GaAs. Moreover, by changing the carrier density and modification of the internal electric field, the PR signals are changed in GaAs.

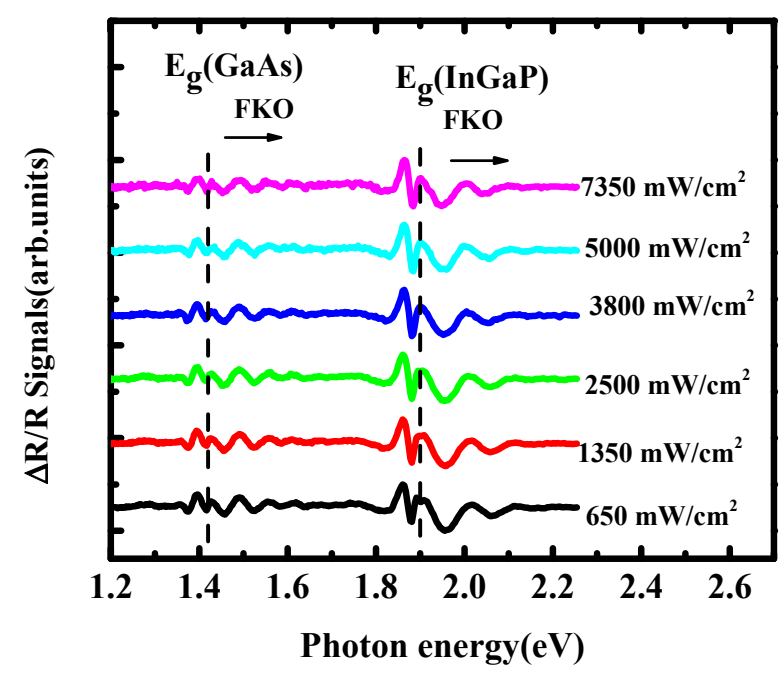

Figure 7. The OBPR spectra of InGaP/GaAs dual junction solar cell as a function of $\mathrm{CW}$ light $(\lambda=404 \mathrm{~nm})$ intensity $\left(\mathrm{I}_{\mathrm{OB}}\right)$ at $300 \mathrm{~K}$.

As we could see by increasing the bias light intensity $\left(\mathrm{I}_{\mathrm{OB}}\right)$, the $\mathrm{FKO}$ oscillations and PR amplitude were changed. This indicated that an increase in the photogenerated carrier density decreases the interface electric fields [12]. The photogenerated carriers create the induced electric field in the opposite direction of the built-in electric field. Therefore, with an increment of the optical bias light intensity, there will be an increase in the generated carriers, resulting in the internal electric field decrement. Figure 8 shows the internal electric field as a function of the $\mathrm{CW}$ light intensity $\left(\mathrm{I}_{\mathrm{OB}}\right) . \mathrm{F}_{\mathrm{pn}}$ is reduced in $\mathrm{InGaP}$ and $\mathrm{GaAs}$ due to the photovoltaic effect [17].

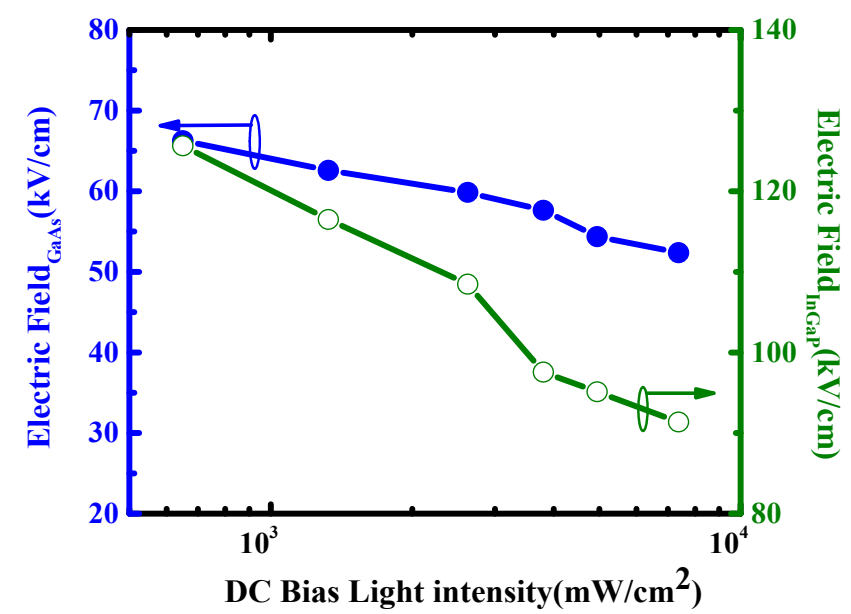

Figure 8. The internal electric field $\left(\mathrm{F}_{\mathrm{pn}}\right)$ of $\mathrm{InGaP}$ (open circle) and $\mathrm{GaAs}$ (solid circle) versus the $\mathrm{CW}$ light intensity $\left(\mathrm{I}_{\mathrm{OB}}\right)$ at $300 \mathrm{~K}$.

We compare the electrical bias voltage and optical bias light intensity-dependent internal electric fields of GaAs SC in Figure 9 to examine how the tunnel junction affects carrier movement in dual junction structure. The pump beam intensity is constant, and therefore, the effect of that is the same in both measurement conditions. In electrically biased photoreflectance, the internal electric field's decrement indicates that the decrement of the potential barrier affects internal electric fields. In these measurements, the $532 \mathrm{~nm}$ laser was used as an excitation source that generates the carriers in both cells, has the 
constant intensity during the measurements, and the carrier's movement effect is constant. Therefore, the variation of potential affects the carrier movement and changed the internal electric field.

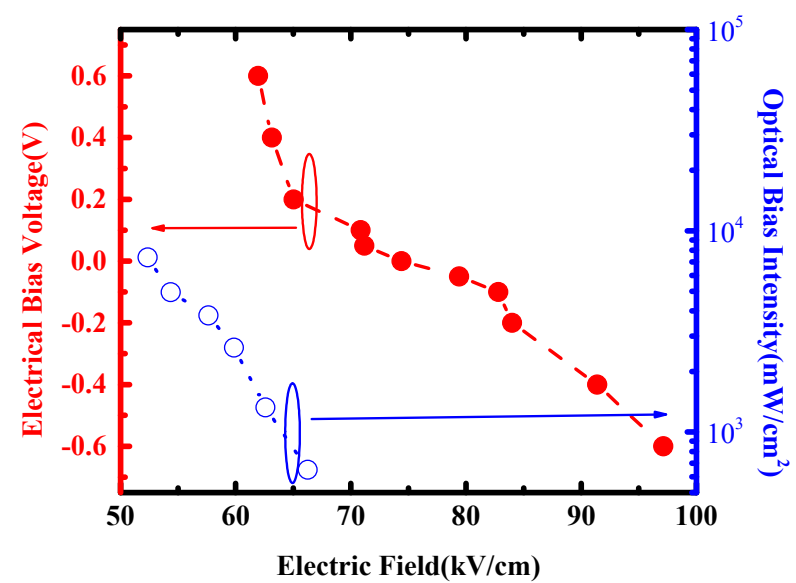

Figure 9. (Color online) Comparison of the change in the GaAs p-n junction's electric field due to electrical (solid circles) and optical (open circles) biases.

Figure 9 shows that $\mathrm{F}_{\mathrm{pn}}$ strength measured under optical bias illumination is lower than $\mathrm{F}_{\mathrm{pn}}$ measured under applying a bias voltage. The pump beam's intensity keeps constant intensity, and therefore the photogenerated carrier density remains constant in each cell. The $404 \mathrm{~nm}$ laser, which is used as the CW light, has energy higher than InGaP and is mostly absorbed by it, and some part of photons can reach the GaAs. The less measured internal electric field strength under optical bias beam illumination describes that in addition to the pump beam effect, the photogenerated carrier density is also changed in GaAs cell even though the bias light has not been absorbed.

As described previously, in the PR measurement, the strength of $F_{p n}$ is proportional to the period of the FKO's, and the period of oscillation is affected by the carrier density variation. Therefore, the results show that the carrier in the GaAs is changed. In contrast, the tunnel junction exported the carrier from one cell to another cell. The photogenerated carriers by the CW light in the top cell pass through the tunnel junction. This indicates that the holes in the p- side of the InGaP pass through the tunnel junction and reach the n-side of GaAs and recombine with the electrons in the region [18]. Changing the carrier density has an effect on the FKO oscillation period and electric field. Thus, the internal electric field in the GaAs is reduced due to the photovoltaic effect.

Therefore, understanding the carrier movement in the dual junction solar cell is important to investigate these structures' current mismatching. In the dual junction structure, the cell which has the lowest current limits the cell current. An investigation of the carrier variation and movement by applying $\mathrm{CW}$ light with different energy is the method to explain the current mismatching in the dual junction solar cells. Therefore, to study the current mismatching, the photovoltaic effect was investigated by optically biased photoreflectance (OBPR) and electrically biased photoreflectance (EBPR) measurements. These spectra are sensitive to the electric field, and the Franz-Keldysh oscillation and the amplitude of the spectrum are changed.

The shape and amplitude of the spectra are changed when the CW light illuminates the SC. The internal electric fields are affected by the carrier density variation, and the variation of the internal electric field is reflected in the spectra and FKO oscillations. The internal electric field is decreased in both cells due to the photovoltaic effect. When the internal electric field changes very small under light illumination, the cell was saturated, and the number of carriers reached equilibrium and not changed more. Therefore, the current in this cell is the lowest. We can say this cell can control and limit the current in the dual junction SC. Based on the results, the internal electric field of InGaP may have the lowest 
changed under illuminated light. Thus, this cell can control and limit the current in the InGaP/GaAs dual junction SC. In addition to improving our result, we will use the I-V curve measurements under illuminated light with performing different wavelengths. In the future, the current mismatching will study in more detail, and we will use the optically biased ER and PR measurements.

\section{Conclusions}

The EBPR and OBPR spectrum measurements were carried out to investigate the tunnel junction effect in the InGaP/GaAs dual junction solar cell. The built-in electric field and its dependence on the external-bias voltage and external CW light were evaluated by the FFT analysis of the FKO in the PR spectrum.

In the EBPR measurements, the pump beam intensity was fixed, and the internal electric field decreased by increasing the DC bias voltage due to the decrement of the potential barrier. In the OBPR results of InGaP and GaAs cells, measurements and the internal electric field decreased due to the photovoltaic effect of carriers generated by the CW light. The OBPR signals are related to the absorption of a CW light. The bias light with energy higher than InGaP is absorbed, and the PR spectra are affected by the photogenerated carriers. Furthermore, the carriers modify the internal electric field in InGaP. Variation of the internal electric field in the GaAs with respect to the $\mathrm{CW}$ light intensity states that the electric field is also modified in GaAs by the carriers generated in the InGaP from the CW light.

More decrement of the internal electric field measured in the OBPR method compared to EBPR indicates that the optical CW light adds a factor. The carrier's density is changed owing to the tunnel effect. The photogenerated carrier in the InGaP moved to the GaAs cell through the tunnel junction and affected the internal electric field in this cell. The tunnel junction plays an essential role in improving the conversion efficiency and current matching in the dual junction solar cells. Additionally, the concentrator multi-junction solar cell with high efficiency has been used in recent years. Therefore, by optimizing the photogenerated carrier's movement through the tunnel junction and improving the current mismatching, we can study the concentrator multi-junction solar cells to getting high efficiency. We will also discuss the other methods for investigating the current mismatching in the multi-junction solar cells, like the photoluminescence and external quantum efficiency measurements.

Author Contributions: “Conceptualization, S.S. and J.S.K. and H.-J.J.; methodology, S.S. and J.S.K. and H.-J.J. and S.J.L. (Sang Jo Lee) and S.J.L. (Sang Jun Lee) and Y.K.; software, S.S.; validation, J.S.K. and H.-J.J.; formal analysis, S.S.; investigation, S.S. and J.S.K.; resources; data curation, S.S. and J.S.K.; writing—original draft preparation, S.S.; writing—review and editing, S.S.; visualization, S.S., J.S.K. and H.-J.J. and S.J.L. (Sang Jo Lee), supervision, J.S.K.; project administration, J.S.K.; funding acquisition, J.S.K. All authors have read and agreed to the published version of the manuscript.

Funding: This research was supported by the National Research Foundation of Korea (NRF) grant funded by the Korea government (NRF-2018R1D1A3B07050824). This work was supported by the National Research Foundation of Korea (NRF) grant funded by the Korea government (NRF2018M3A7B4069996). The Priority Research Centers Program supported this study through the National Research Foundation of Korea (NRF) funded by the Ministry of Education (2014R1A6A1031189).

Institutional Review Board Statement: Not applicable

Informed Consent Statement: Not applicable.

Data Availability Statement: Not applicable.

Conflicts of Interest: The authors declare no conflict of interest. 


\section{References}

1. Canovas, E.; Fuertes Marron, D.; Martí, A.; Luque, A.; Bett, A.W.; Dimroth, F.; Philipps, S.P. Photoreflectance analysis of a GaInP/GaInAs/Ge multijunction solar cell. Appl. Phys. Lett. 2010, 97, 203504. [CrossRef]

2. Amine, A.; Mir, Y.; Zazoui, M. Modelling of dual-junction solar cells including tunnel junction. Adv. Condens. Matter Phys. 2013, 2013, 546362. [CrossRef]

3. Shen, D.S.; Schropp, R.E.; Chatham, H.; Hollingsworth, R.E.; Bhat, P.K.; Xi, J. Improving tunneling junction in amorphous silicon tandem solar cells. Appl. Phys. Lett. 1990, 56, 1871-1873. [CrossRef]

4. Takamoto, T.; Ikeda, E.; Kurita, H.; Ohmori, M. Over 30\% efficient InGaP/GaAs tandem solar cells. Appl. Phys. Lett. 1997, 70, 381-383. [CrossRef]

5. Khan, A.; Yamaguchi, M.; Takamoto, T. Performance of single-junction and dual-junction InGaP/GaAs solar cells under low concentration ratios. Appl. Phys. Lett. 2004, 85, 3098-3100. [CrossRef]

6. Wang, Z.H.; Pan, S.H.; Li, L.W.; Lin, H.K.; Gong, Y.S. Effect of chemical treatment on the photoreflectance of semi-insulating GaAs. Phys. Lett. A 1994, 192, 141-147. [CrossRef]

7. Pollak, F.H.; Shen, H. Modulation spectroscopy in superlattices. Superlattices Microstruct. 1989, 6, 203-212. [CrossRef]

8. Wang, R.; Jiang, D. Photoreflectance spectroscopy of semi-insulating GaAs. J. Appl. Phys. 1992, 72, 3826-3828. [CrossRef]

9. Nayak, P.P.; Dutta, J.P.; Mishra, G.P. Efficient InGaP/GaAs DJ solar cell with double back surface field layer. Eng. Sci. Technol. Int. J. 2015, 18, 325-335. [CrossRef]

10. Katayama, R.; Kuroda, M.; Onabe, K.; Shiraki, Y. Electrically biased photoreflectance study of cubic GaN/GaAs (001) heterointerface. Physica Status Solidi (c) 2003, 0, 2597-2601. [CrossRef]

11. Lee, S.J.; Sohn, C.W.; Jo, H.J.; Kim, J.S.; Noh, S.K.; Choi, H.; Leem, J.Y. Temperature dependence of the photovoltage from Franz-Keldysh oscillations in a GaAs p+-in+ structure. J. Korean Phys. Soc. 2015, 67, 916-920. [CrossRef]

12. SaeidNahaie, S.; Entezar, S.R.; Naghshara, H.; Jo, H.J.; Kim, J.S.; Kim, Y.; Lee, S.J. Investigation of the incident light intensity effect on the internal electric fields of GaAs single junction solar cell using bright electroreflectance spectroscopy. Curr. Appl. Phys. 2020, 20, 145-149. [CrossRef]

13. Neamen, D.A. Semiconductor Physics and Devices: Basic Principles; McGraw-Hill: New York, NY, USA, 2012.

14. So, M.G.; Jo, H.J.; Kim, J.S.; Lee, S.J.; Noh, S.K.; Leem, J.Y. Evaluation of the photo-generated carrier density of GaAs solar cells by using electrical and optical biased electroreflectance spectroscopy. J. Korean Phys. Soc. 2015, 67, 723-727. [CrossRef]

15. Green, R.J.; Ali, N.; Chol, A.M. Optical performance of the silicon heterostructure switch. IEEE Trans. Electron. Devices 1989, 36, 25-30. [CrossRef]

16. Van Zeghbroeck, B. Principles of Electronic Devices; University of Colorado: Denver, CO, USA, 2011.

17. Leem, J.W.; Lee, Y.T.; Yu, J.S. Optimum design of InGaP/GaAs dual-junction solar cells with different tunnel diodes. Opt. Quantum Electron. 2009, 41, 605-612. [CrossRef]

18. Takamoto, T.; Yumaguchi, M.; Ikeda, E.; Agui, T.; Kurita, H.; Al-Jassim, M. Mechanism of Zn and Si diffusion from a highly doped tunnel junction for InGaP/GaAs tandem solar cells. J. Appl. Phys. 1999, 85, 1481-1486. [CrossRef] 